\title{
Development of a Master Slave Combined Manipulator for Laparoscopic Surgery
}

\author{
Functional Model and Its Evaluation
}

\author{
Makoto Jinno ${ }^{1}$, Nobuto Matsuhira ${ }^{1}$, Takamitsu Sunaoshi ${ }^{1}$ Takehiro Hato ${ }^{1}$, \\ Toyomi Miyagawa ${ }^{1}$, Yasuhide Morikawa ${ }^{2}$, Toshiharu Furukawa ${ }^{2}$, Soji Ozawa ${ }^{2}$, \\ Masaki Kitajima ${ }^{2}$, and Kazuo Nakazawa ${ }^{3}$ \\ ${ }^{1}$ Corporate R\&D Center, Toshiba Corporation \\ 1, Komukai Toshiba-Cho, Kawasaki-Ku, Kawasaki 212-8582, Japan \\ ${ }^{2}$ Department of Surgery, Keio University \\ 35, Shinano-machi, Shinjyuku-ku, Tokyo 160-8582, Japan \\ ${ }^{3}$ Department of System Design Engineering, Keio University \\ 3-14-1 Hiyoshi, Kohoku-ku, Yokohama 223-8522, Japan
}

\begin{abstract}
Minimally invasive surgery helps patients by accelerating postoperative recovery. However, its application is impeded because it is necessary for the surgeons performing such surgery to possess surgical skills of a high order. Thus, for laparoscopic surgery, a master slave combined manipulator (MCM) has been proposed that enhances the surgeon's skill. The master grip and the slave hand with wrist joints are combined through the manipulator body, and a surgeon can perform the operation near to the patient. The slave hand is controlled by the master grip electrically and its position is directly controlled by the surgeon. The prototype model of the MCM has been developed and the function of the MCM has been verified by basic evaluation tests, and the MCM has been applied in an animal experiment. This paper describes the basic performance of the MCM.
\end{abstract}

\section{Introduction}

Laparoscopic surgery and other types of minimally invasive surgery (MIS) have entered widespread use in recent years. In laparoscopic surgery, a surgeon performs a surgical operation by using forceps through a few holes in the patient's abdomen, each hole being about $10 \mathrm{~mm}$ in diameter, while monitoring the image captured by a laparoscope. Owing to the small incision, the patient can recover earlier and suffers less pain, and the medical cost is reduced. Thus, laparoscopic surgery is highly advantageous for the patient. However, it is necessary for the surgeon performing such surgery to possess surgical skills of a high order. One of the main reasons is the lack of degrees of freedom for the free motion of the forceps. Applying techniques from robotics, we add wrist joints to the conventional forceps, and thus construct a robotic tool. The manipulator is able to enhance the surgeon's skill, reduce the demands imposed on the surgeon by laparoscopic surgery, and greatly contribute to the quality of the life (QOL) of the patient. Thus, a master slave combined manipulator has been newly developed. It was evaluated by the basic experiments such as suturing and 
ligaturing using a phantom model and applied in an animal experiment. As a result, the validity of the surgical manipulator was verified.

\section{Concept of a Master Slave Combined Manipulator for Laparoscopic Surgery}

In laparoscopic surgery, the gripper's orientation of the forceps cannot be freely changed during the operation. However, it is highly desirable that the surgeon should be able to move the tip of the forceps as if it were his/her own hand. For that purpose, a technique employed for tele-operated robots that is analogous to the master slave manipulator is applicable. Surgical robots developed so far [1]-[6] are based on the same concept as that of the conventional master slave manipulator; that is, the slave arm is controlled from the master arm at an operational console separated from the patient. In this concept, the master arm and the slave arm can be designed independently, resulting in a more suitable design of the master arm for the surgeon in terms of operability, respecting such items as joint alignment and arm size. However, such a master slave manipulator makes a system mechanically and electrically complicated from the viewpoints of safety and maintenance. On the other hand, human/robot cooperative systems and advanced instruments have been variously developed [7]-[11]. Our approach is to develop a robotic tool, applying a master slave manipulator for an intuitive operation, which is able to be used near to a patient in combination with conventional surgical tools. Thus, a master slave combined manipulator is proposed for the robot forceps application [12] as shown in Fig.1. The manipulator should be a simple structure, highly reliable, and safe, and the surgeon should perform the operation near to the patient so as to be able to cope with any emergency, such as a sudden hemorrhage. Furthermore, the manipulator will be used for applications in general surgery rather than in cardiac surgery.

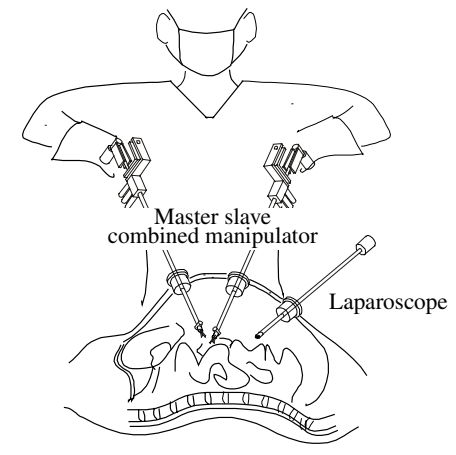

Fig.1. Image of laparoscopic surgey using master slave combined manipulators

\section{Design of the Functional Model of a Master Slave Combined Manipulator}

Fig.2 shows the structure of the developed surgical manipulator for laparoscopic surgery. The wrist joints are added to the forceps and they are operated from the grip in 
the same way as for a master slave operation. Since the master grip (control device) and the slave hand (operation device) are combined through the manipulator body, it is called a master slave combined manipulator (MCM). According to this concept, a surgeon will acquire greater skill in use of the MCM with practice, because it is a tool. In the previous paper [12], the support mechanism was proposed to hold the MCM in any position and compensate for its deadweight. Here, the functional model of the MCM without the support mechanism has been developed.

\subsection{Structure of the Manipulator}

A manipulator needs six degrees of freedom (DOF) in order to be deployed in a desired position and orientation in space. Since the tip position and the roll movement of the forceps are operated by the surgeon directly, the forceps needs more two DOF as a manipulator. Thus, two DOF are to be added to the forceps. Here, these two DOF are considered for performing the suturing task. In suturing, a semicircular needle is used generally. The needle has to be moved along a circular trajectory as shown in Fig.3. In the case that the tip joint of the MCM is roll axis, it is very easy to realize such a trajectory for any posture. However, if the tip joint is yaw axis or pitch axis, coordinated operation for orientational axes is required to operate along the circular trajectory. In the case of the MCM, such an operation is very difficult because of the structure. Therefore, the joint alignment of yaw axis (or pitch axis), roll axis, and a gripper is designed shown in Fig.2.

\subsection{Slave Hand Mechanism}

The slave hand consists of a gripper, roll and yaw axes. These axes are cable-driven by the DC servomotors mounted near the master grip. In order to reduce the number of DOF and decrease the weight of the manipulator, the roll axis around the manipulator body is commonly used for both the master grip and the slave hand. Additionally, the hand open/close motion is realized by the pinching mechanism of the master fingers.

\subsection{Master Grip Mechanism}

The master grip has yaw and roll axes and a gripper in the same order as the slave hand. The three axes of the master grip which roll, yaw and roll axes intersect at a point. In this structure, the operability of the MCM is found to be better in the case that the orientation is separated from the translation mechanically, because tip position does not move when the master grip orientation is changed. If the operation of the master grip orientation had an effect on the position of the slave gripper, precise surgical operation would be difficult. For the same reason, a gimbal mechanism has been widely adopted in previous master manipulators [13]. The master grip and the motor unit are to be separated from the body for the sterilization.

\subsection{Control Method}

The tip position of the MCM is controlled by the surgeon's direct motion and the orientation is determined by electrical signals generated by the master grip. The surgeon is responsible for the large and quick motion of the MCM. This is desirable from the viewpoint of safety. Fig.4 shows the control block diagram. The slave hand is controlled by the unilateral servo control method, i.e., each joint angle of the master grip is detected by the potentiometer and the slave hand is controlled to follow the 
joint angles by the encoders mounted on the servomotors. A notebook computer with an interface unit is used for the controller. By using motors, it would be possible to shift the motion area of the slave hand and change the motion ratio from the master to the slave hand for the improvement of the operability. Basic specifications and photograph of the developed MCM are shown in Table1 and Fig.5, respectively. In particular, the diameter of the manipulator is $12 \mathrm{~mm}$, which is sufficient for conventional laparoscopic surgery.

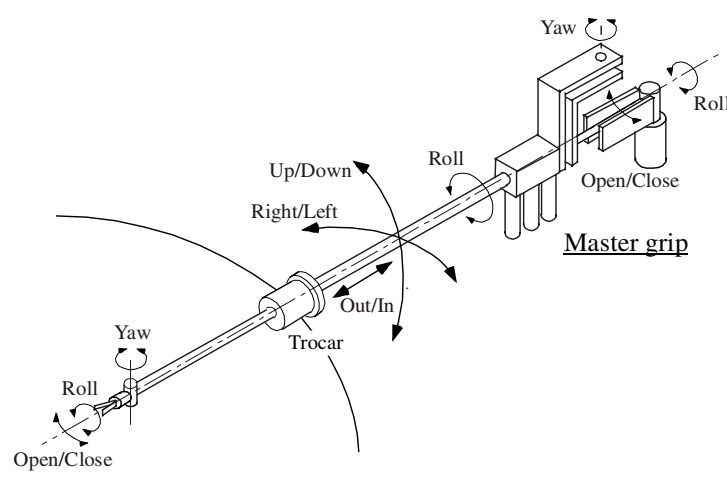

$\underline{\text { Slave hand }}$

Fig. 2. Structure of the MCM

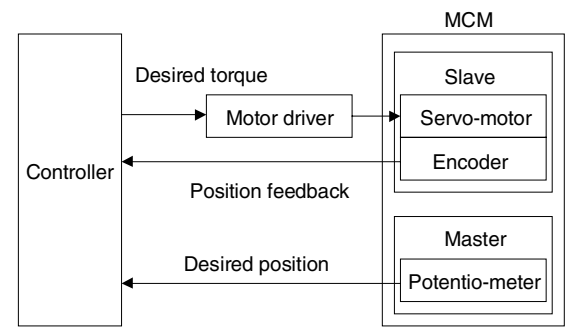

Fig. 4. Control block diagram

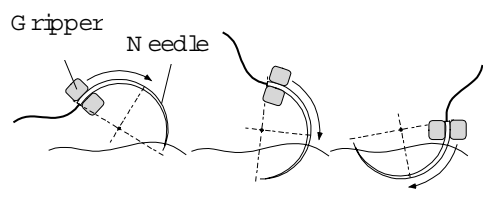

Fig. 3. Suturing task by a circular needle

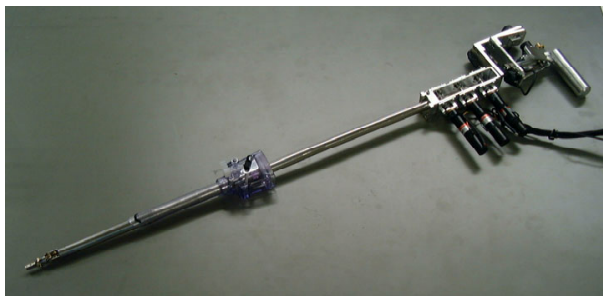

Fig. 5. Photograph of the developed MCM

Table 1. Specifications of the developed MCM

\begin{tabular}{|l|l|l|}
\hline Items & \multicolumn{2}{|c|}{ Specification } \\
\hline \multirow{3}{*}{ Size } & Total Length & $700[\mathrm{~mm}]$ \\
\cline { 2 - 3 } & Pipe diameter & $12[\mathrm{~mm}]$ \\
\hline \multirow{3}{*}{ Weght } & \multicolumn{2}{|c|}{$0.6[\mathrm{~kg}]$} \\
\hline \multirow{3}{*}{ Sotion range } & Yaw & $\pm 90\left[^{\circ}\right]$ \\
\cline { 2 - 3 } & Roll & $\pm 90\left[^{\circ}\right]$ \\
\cline { 2 - 3 } & Gripper & $\pm 30\left[^{\circ}\right]$ \\
& & $(10[\mathrm{~mm}])$ \\
\hline Control & \multicolumn{3}{|c|}{ Unilateral control } \\
\hline \multirow{2}{*}{ Sensor } & Master & Potentiometer \\
\cline { 2 - 3 } & Slave & Encoder \\
\hline
\end{tabular}




\section{Basic Evaluation Experiments for the Functional Model of the MCM}

Basic evaluation tests were performed for the MCM using a phantom model as shown in Fig.6. Here, the phantom model is made of a sponge for a stomach. Suturing and ligaturing tasks were performed to evaluate the position accuracy and operability of the MCM measured by the operation time and position errors. These tasks require dexterous manipulation in laparoscopic surgery.

\subsection{Evaluation Task}

Suturing and ligaturing tasks for evaluation were performed by operators who held the MCM in their right hands and the conventional forceps in their left hands, watching through the image of the laparoscope. The operators were three engineers who developed the MCM. Circular needles with $22 \mathrm{~mm}$ and $25 \mathrm{~mm}$ length were used for the evaluation task.

\subsection{Operation Time for Suturing and Ligaturing}

The operation time was measured from the insertion of the needle at any point to the completion of the ligature, four times per operator. In the ligaturing task, a surgical knot was used such as three single knots including double loop at the first knot. The result is shown in Fig.7 by box plots. The operation times shown is for suturing, knot1 , knot-2, knot-3, and total time, respectively. The average time is about $155 \mathrm{sec}$ for the task. It was almost equal to the time using a conventional forceps instead of the MCM.

\subsection{Operation Time for Continuous Suturing}

Five continuous suturing tasks for horizontal and vertical directions were performed twice for three operators in Fig.8. The operation time and position accuracy of the continuous suturing task is shown in Fig.9. Fig.11 (1) shows the results. The phantom has marked points for insertion and penetration at suturing. The operation time contains changing time of the needle's posture for a suture. The error shows the position error between the marked point and actual insertion point. The average error was within $1 \mathrm{~mm}$ and one suturing time was about $65 \mathrm{sec}$ in the continuous suturing in Fig.9. Angles of yaw and roll axes during the operation are shown in Fig.10. Note that roll axis was used effectively rather than the yaw axis in the suturing. Thus, the roll axis worked well for suturing.

\subsection{0-Degree Suturing}

One feature of the MCM is that suturing can be executed from any direction. This is impossible using a conventional forceps. To verify this feature clearly, 360-degree suturing was performed as shown in Fig.11 (2). In the horizontal plane, the task was almost realized. Although conventional forceps can move only around the axis of the forceps' body, the MCM can suture within the +-90 directions using the yaw axis. Rotating the tip roll axis of the slave hand, the needle is moved along the circular trajectory smoothly. The MCM was verified to be useful for suturing tasks. 


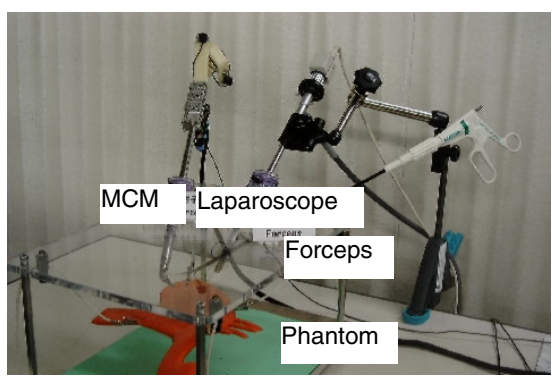

Fig. 6. Experimental setup

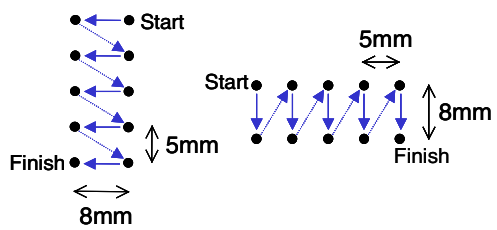

(1) Horizontal

(2) Vertical

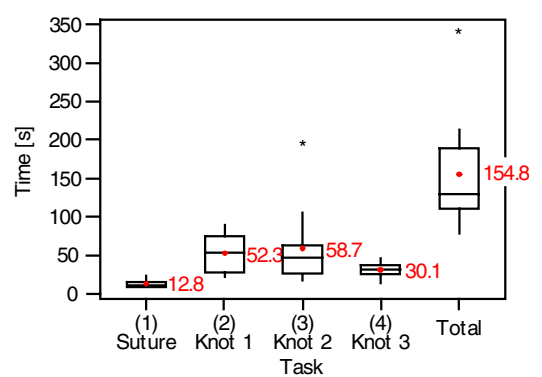

Fig. 7. Suturing and ligaturing time

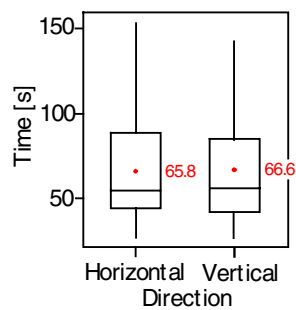

(1) Suturing time

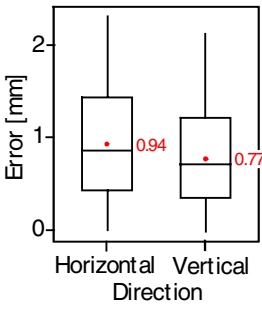

(2) Suturing error

Fig. 8. Continuous suturing task

Fig. 9. Continuous suturing time and position error

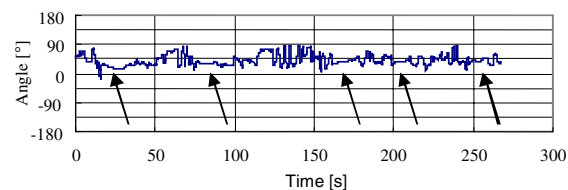

(1) Yaw angle

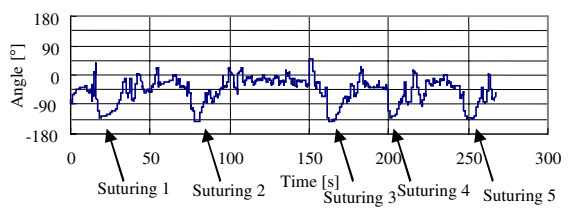

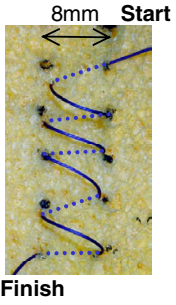

(1) Horizontal direction

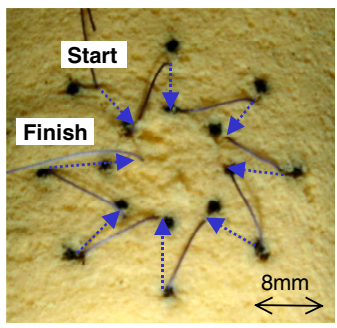

(2) Various direction

(2) Roll angle

Fig. 10. Joint angles of continuous suturing task

Fig. 11. Suturing results

\section{Preliminary Animal Experiment}

The preliminary animal experiment was performed before the basic evaluation tests in Chapter 4. The purpose of the animal experiment is to identify any problems concerning the MCM. After the following experiments, gripping force and working area of the MCM were improved. The MCM was partially applied for the Nissen surgery involving suturing and ligaturing tasks. The surgeon practiced for an hour before the 
operation. In the surgical operation, the surgeon manipulated the MCM using his right hand and the conventional forceps using his left hand, watching the monitor from the laparoscope as shown in Fig. 12.

\subsection{Suturing Task}

Fig.13 (1)-(4) show the suturing task performed using the MCM. During the penetration, it was confirmed that the yaw axis was not so moved and the roll axis of the slave hand was mainly used.

\subsection{Ligaturing Task}

Fig.13 (5)-(8) show the ligature task performed using the MCM. The ligature task consists of four knots; the MCM winds the thread twice around the conventional forceps, the forceps winds around the MCM once, and MCM winds around the forceps once. The MCM changes the posture of the slave hand depending on the conditions, such as, the case that the MCM winds the thread around the forceps, the case that the forceps winds around the MCM, and the case that the needle is passed between the MCM and forceps. In the operation, there are many requirements to change the posture of the MCM.

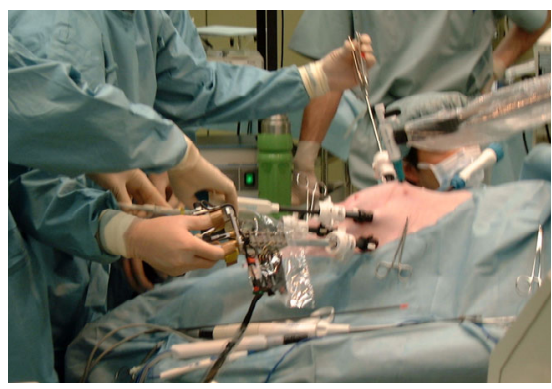

Fig. 12. Overview of the animal experiment
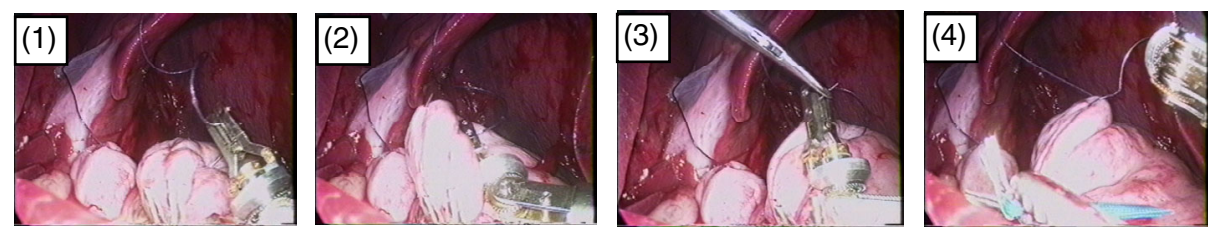

(a) Suturing task
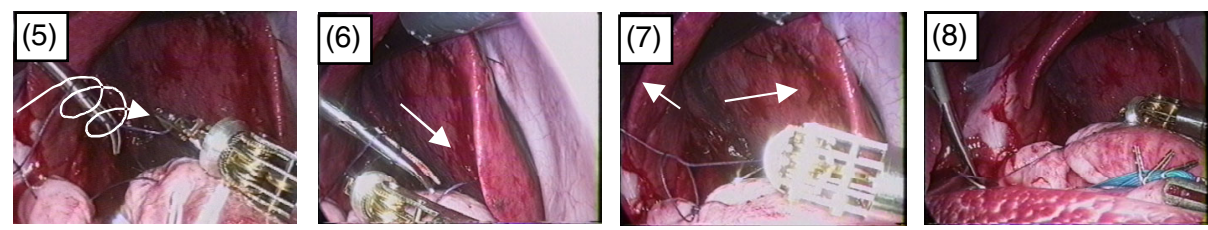

(b) Ligaturing task

Fig. 13. Suturing and ligaturing task performed using the MCM 


\section{Conclusion}

The functional model of the master slave combined manipulator (MCM), a robotic forceps for laparoscopic surgery, has been developed. The validity of the MCM was confirmed by the basic evaluation experiments and the animal experiment as follows.

- Suturing from any direction that conventional forceps cannot perform is possible - Suturing error is about $1 \mathrm{~mm}$ towards marked points

Next, with a view to proceeding to clinical tests, the operability and the reliability of the MCM should be improved, taking sterilization into consideration.

This development is carried out as part of a project concerning an advanced support system for endoscopic and other minimally invasive surgery by the New Energy and Industrial Technology Development Organization (NEDO) for 2000-2004.

\section{References}

1. http://www.computermotion.com

2. http://www.intuitivesurgical.com

3. A.J.Madhani, G.Niemeyer, J.K.Salisbury: The Black Falcon: A Teleoperated Surgical Instrument for Minimally Invasive Surgery, Proc. 1998 IEEE/RSJ Int. Conf. IROS (1998) 936-944

4. K.Ikuta, M.Nokata, S.Aritomi: Hyper Redundant Active Endoscope for Minimally Invasive Surgery, Journal of Robotics Society of Japan, Vol.16, no.4 (1998) 569-575 (in Japanese)

5. M.C.Cavusoglu, F.Tendick, M.Cohn, S.S.Sastry: A Laparoscopic Telesurgical Workstation, IEEE Trans. Robotics and Automation, Vol.15, No.4 (1999) 728-739

6. M.Mitsuishi, S.Tomisaki, T.Yoshidome, H.Hashizume, K.Fujiwara: Tele-micro-surgery system with intelligent user interface, Proc. IEEE Int. Conf. on Robotics and Automation, vol.2 (2000) 1607-1614

7. H.Kazerooni, Human/robot interaction via transfer of power and information signals Part I: Dynamics and Control analysis, Proc. IEEE Int. Conf on Robotics and Automation (1989) $1632-1640$

8. M.O.Schurr: Robotic Devices for Advanced Endoscopic Surgical Procedures: An Overview, Journal of Robotics Society of Japan, Vol.18, No.1 (2000) 16-19

9. R.Kumar, P.Berkelman, P.Gupta, A.Barnes, P.Jensen, L.L.Whitcomb, R.H.Taylor: Preliminary Experiments in Cooperative Human/Robot Force Control for Robot Assisted Microsurgical Manipulation, Proc. IEEE Int. Conf. on Robotics and Automation (2000) 610-617

10. R.Nakamura, T.Oura, E.Kobayashi, I.Sakuma, T.Dohi, N.Yahagi, T.Tsuji, D.Hashimoto, M.Shimada, M.Hashizume: Multi-DOF Forceps Manipulator System for Laparoscopic Surgery - Mechanismminiaturized \& Evaluation of New Interface -, Proc. 4th MICCAI2001 (2001) 606-613

11. S.Shimachi, Y.Hakozaki, A.Oguni, A.Hashimato: Weld Knotting and Contact Force Sensing for Laparoscoppic Surgery, Proc. 18th Annual Conf. of the Robotics Society of Japan (2000) 849-850 (in Japanese)

12. N.Matsuhira, H.Hashimoto, M.Jinno, T.Miyagawa, K.Nambu, Y.Morikawa, T.Furukawa, M.Kitajima, K.Nakazawa: Development of a Manipulator for Laparoscopic Surgery - Conceptual model of master slave combined manipulator and its evaluation -, Proc. 32nd ISR (2001) 630-635

13. N.Matsuhira, H.Bamba and M.Asakura: The development of a general master arm for teleoperation considering its role as a man-machine interface, Advanced Robotics, Vol.8, No.4 (1994) 443-457 